\title{
Effective treatment of a patient with stage IV ovarian cancer: A case report
}

\author{
ZHEN HUANG $^{1}$, HUA YAN ${ }^{2}$, DEVENDRA CHAVAN ${ }^{1}$, ZENG YUAN $^{1}$, \\ XINGSHENG YANG ${ }^{1}$, YOUZHONG ZHANG ${ }^{1}, \mathrm{KUN} \mathrm{SONG}^{1}$ and BEIHUA KONG ${ }^{1}$ \\ ${ }^{1}$ Department of Obstetrics and Gynecology, Qilu Hospital of Shandong University; \\ ${ }^{2}$ Department of Spleen and Stomach Diseases, Affiliated Hospital of Shandong University of \\ Traditional Chinese Medicine, Jinan, Shandong 250012, P.R. China
}

Received November 6, 2016; Accepted September 1, 2017

DOI: $10.3892 / \mathrm{ol} .2017 .7285$

\begin{abstract}
The present case report describes a patient with stage IV ovarian cancer who survived for 9 years without achieving a complete remission since the first relapse. The patient's long-term survival may be ascribed to the slow growth of the cancer cells, limited metastasis and favorable responses to the treatments received. The patient manifested initially with malignant pleural effusion and was not able to tolerate surgical treatment. However, the disease was well-controlled using various treatments, including neoadjuvant chemotherapy, interval cytoreductive surgery and post-operative long-term chemotherapy. Management of ovarian cancer is most effective when tailored to the individual needs of the patient, maximizing its efficacy and prolonging the patient's survival rate. The present case may offer useful insight into the clinical management of stage IV ovarian cancer
\end{abstract}

\section{Introduction}

Among all types of female reproductive system cancer, ovarian cancer has the second highest incidence rate and the highest mortality rate (1). At the time of diagnosis, $75 \%$ of patients are at an advanced stage (III or IV) (2). The 5-year survival rate decreases rapidly between stages I and IV (3). Currently, no effective methods exist for the screening and early diagnosis of ovarian cancer. The majority of patients have already reached advanced stages at diagnosis, resulting in a poor prognosis (2). The 5-year relative survival rate ranges from between 30 and $40 \%$ at stage III to $<10 \%$ at stage IV (2).

Correspondence to: Dr Kun Song, Department of Obstetrics and Gynecology, Qilu Hospital of Shandong University, 107 Wenhuaxi Road, Jinan, Shandong 250012, P.R. China

E-mail: songkun2001226@163.com

Key words: interval cytoreductive surgery, neoadjuvant chemotherapy, post-surgery chemotherapy, ovarian neoplasms, treatment
Nevertheless, practicing personalized medicine catering to individual responses and a timely follow-up may achieve a satisfactory therapeutic outcome in certain advanced-stage patients. In the present case report, the step-by-step management protocol followed for a patient with stage IV ovarian cancer who survived for $>9$ years is described. As it is rare for a patient with advanced-stage ovarian cancer to demonstrate such favorable prognosis, it is hoped that the treatment protocol may be of assistance to clinicians encountering a similar case. The patient provided written informed consent for the publication of the present study.

\section{Case report}

The patient was a 48 -year-old female who was admitted to the pulmonary department of Qilu Hospital of Shandong University (Jinan, China) due to a severe irritating cough and chest tightness in July 2004. The patient's mother had a history of tuberculosis. Computed tomography (CT) scan results revealed pleural effusion in the right lung and pericardial effusion with mild ascites. The suspected diagnosis upon admission was tuberculous pleurisy. Thoracocentesis yielded $1,000 \mathrm{ml}$ yellow serous exudate, which tested negative for Mycobacterium tuberculosis and malignant cells. Abdominal paracentesis also revealed similar results. Physical examination revealed a palpable mass in the recto-uterine pouch. After 2 weeks of conservative therapy, the patient's situation was slightly improved. However, at the end of the third week, the patient's condition began to deteriorate. Lumps appeared on the right arm and right supraclavicular region. Enhanced CT scan results indicated a number of enlarged lymph nodes surrounding the right side of the thoracic entrance to the mediastinum, multiple swollen cervical lymph nodes on the right side and right internal jugular vein embolus, with bilateral pleural effusion and ascites. CT-guided pelvic mass biopsy was then performed to clarify the diagnosis. Histopathological examination revealed papillary adenocarcinoma originating from the reproductive system. As the patient was not able to tolerate surgery, neoadjuvant chemotherapy was considered, and four courses of cisplatin and paclitaxel were administered. All the pleural effusion, peritoneal effusion and right internal jugular vein tumor thrombus disappeared. Mediastinal lymph 
Table I. Treatments administered to the patient with Stage IV ovarian cancer.

\begin{tabular}{|c|c|c|c|}
\hline $\begin{array}{l}\text { Time following } \\
\text { diagnosis, months }\end{array}$ & $\begin{array}{l}\text { CA125 level prior } \\
\text { to treatment, } \mathrm{U} / \mathrm{ml}\end{array}$ & Treatment & $\begin{array}{l}\text { CA125 level following } \\
\text { treatment, } \mathrm{U} / \mathrm{ml}\end{array}$ \\
\hline 1 & $>900.0$ & Cisplatin + paclitaxel, 4 cycles & 157.00 \\
\hline 4 & 157.0 & Interval cytoreductive surgery & 86.00 \\
\hline 4 & 86.0 & Docetaxel + carboplatin, 3 cycles & 35.00 \\
\hline 24 & 41.6 & Liposomal doxorubicin + trabectedin, 4 cycles & 35.00 \\
\hline 43 & 120.0 & Docetaxel + carboplatin, 8 cycles & 35.00 \\
\hline 57 & 304.6 & Topotecan, 2 cycles; carboplatin + Taxol, 1 cycle & 452.10 \\
\hline 58 & 482.5 & Oxaliplatin + vinorelbine, 6 cycles & 20.35 \\
\hline 68 & 186.5 & $\begin{array}{l}\text { Gemcitabine + campecitabene, } 2 \text { cycles; } \\
\text { gemcitabine + cyclophosphamide, } 4 \text { cycles }\end{array}$ & 84.04 \\
\hline 73 & 105.0 & Epirubicin + vindesine, 3 cycles & 225.30 \\
\hline 76 & 238.1 & Nedaplatin + vinorelbine, 6 cycles & 35.00 \\
\hline 84 & 166.7 & Lobaplatin + vinorelbine, 7 cycles & 35.00 \\
\hline 94 & 262.5 & Lobaplatin + irinotecan, 4 cycles & 154.70 \\
\hline 100 & 321.2 & $\begin{array}{l}\text { Nedaplatin + irinotecan, } 2 \text { cycles Lobaplatin }+ \\
\text { nedaplatin + irinotecan, } 1 \text { cycle }\end{array}$ & 234.20 \\
\hline 105 & $2,222.0$ & Symptomatic treatment & \\
\hline 109 & & Patient succumbed & \\
\hline
\end{tabular}

CA125, cancer antigen 125 .

nodes and pelvic mass were also markedly decreased in size. The patient's physical condition improved and so interval cytoreductive surgery was performed. Routine postoperative pathological examination identified undifferentiated carcinoma on the left ovary with right ovary metastasis, without any metastasis to the pelvic lymph nodes. Postoperative docetaxel plus carboplatin chemotherapy was administered for 3 cycles and serum cancer antigen 125 (CA125) level returned to normal. During a follow-up 19 months after surgery, CT revealed a pelvic mass $2 \mathrm{~cm}$ in diameter and the serum CA125 level increased to $41.6 \mathrm{U} / \mathrm{ml}$. A total of 4 cycles of liposomal doxorubicin- plus trabectedin-based second-line chemotherapy were administered, and CA125 decreased again to normal levels. CT scanning 7 months later revealed a mass in the left anterior region of the sigmoid colon and the CA125 level once again increased to $120 \mathrm{U} / \mathrm{ml}$. Third-line docetaxel and carboplatin chemotherapy was administered for 8 cycles and the CA125 level subsequently decreased to $9.45 \mathrm{U} / \mathrm{ml}$. On follow-up (1 year), the patient's serum CA125 level was increased again to $304.6 \mathrm{U} / \mathrm{ml}$ and ultrasonography revealed a hypoechoic solid nodule in the pelvis. Fourth-line topotecan-based chemotherapy was administered for 2 cycles, then changed to 1 cycle of carboplatin and paclitaxel due to marked toxicity. The patient's CA125 level gradually increased to $452.10 \mathrm{U} / \mathrm{ml}$ during chemotherapy. The patient's condition was not able to be controlled with the current regimen as a result of multidrug resistance. Treatment was changed to a combination of oxaliplatin and vinorelbine for 6 cycles, with CA125 levels subsequently decreasing to $20.35 \mathrm{U} / \mathrm{ml}$. The patient was admitted 5 months later due to relapse. The patient was then administered 2 cycles of gemcitabine and capecitabene and 4 cycles of gemcitabine and cyclophosphamide.

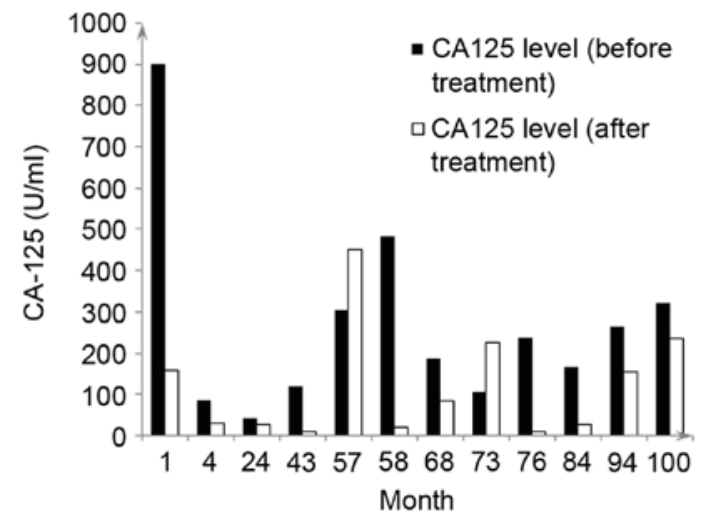

Figure 1. Levels of CA125 prior to and following treatment. CA125, cancer antigen 125 .

The CA125 level was $84.05 \mathrm{U} / \mathrm{ml}$ following this round of chemotherapy. The patient relapsed again 1 month later, and an epirubicin and vindesine regimen was administered for 3 cycles. Since January 2011, 6 cycles of a nedaplatin and vinorelbine combination regimen were administered. Upon completion of this course, the level of CA125 had decreased to $8.93 \mathrm{U} / \mathrm{ml}$. The patient then attained a stable condition and was monitored carefully during follow-up.

The patient's CA125 levels once again increased to 166.70 U/ml 1 month later. The patient was therefore administered 6 cycles of lobaplatin and vinorelbine. The patient's CA125 level was measured at $25.69 \mathrm{U} / \mathrm{ml}$ following this course of chemotherapy, until relapse, increasing to 262.50 U/ml after 3 months. After 18 days of treatment with 1 cycle of lobaplatin $(50 \mathrm{mg})$ and irinotecan $(280 \mathrm{mg})$, the 
patient developed severe bone marrow suppression [white

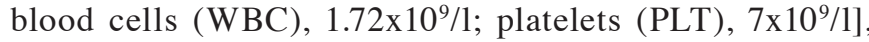
high fever and diarrhea. However, the CA125 levels returned to normal at $28.51 \mathrm{U} / \mathrm{ml}$. Dosage was decreased to $40 \mathrm{mg}$ lobaplatin and $200 \mathrm{mg}$ irinotecan owing to the potential side effects. The CA125 levels were unsatisfactory after 1 month of treatment $(54.49 \mathrm{U} / \mathrm{ml})$, thus irinotecan $(240 \mathrm{mg})$ was added for a further 2 cycles. However, the CA125 levels increased continuously to $86.93 \mathrm{U} / \mathrm{ml}$. CA125 levels were consistently increased after 1 month, therefore treatment was changed to nedaplatin and irinotecan which yielded poor results. The level of CA125 even increased further to $321.2 \mathrm{U} / \mathrm{ml}$. At this stage, an alternative drug regimen was advised, which the patient declined due to high expense. Thus, 1 cycle of lobaplatin was added without any marked alteration in the CA125 levels. Furthermore, the patient presented with severe bone marrow suppression (WBC, $0.4 \times 10^{9} / 1$; PLT, $2 \times 10^{9} / 1$ ) and hemorrhage of the digestive tract, which were managed symptomatically. In June 2013, the patient's CA125 level was revealed to be $2,222 \mathrm{U} / \mathrm{ml}$. The patient was hospitalized 2 days later due to severe gastrointestinal bleeding. Positron emission tomography-CT revealed a recurrent pelvic lesion measuring $9.2 \times 7.2 \times 5.5 \mathrm{~cm}$, involving the rectum. CT revealed bilateral pleural effusion. B-mode ultrasonography revealed multiple solid masses in the liver. Considering the poor outcome and relapse of tumor with metastasis, palliative treatment was offered. The patient was hospitalized again due to severe gastrointestinal hemorrhage in August 2015. The patient's family members declined any further treatment and the patient was discharged having received symptomatic treatment. The patient succumbed 2 months later. All chemotherapy treatments received are summarized in Table I. The CA125 levels of the patient prior to and following treatment are presented in Fig. 1.

\section{Discussion}

Among all types of female reproductive system cancer, ovarian cancer exhibits the second highest incidence rate and the highest mortality rate (1,3). Ovarian cancer in $75 \%$ of patients has already reached an advanced stage at the time of diagnosis (2). In the present case, the patient initially presented with pleural effusion and ascites. Supraclavicular lymph node metastasis indicated that the disease had already progressed to stage IV.

The patient's poor physical condition precluded surgery directly, although a concurrent pelvic mass existed. Neoadjuvant chemotherapy was selected as the first-line therapy based on histological evidence. Histopathology confirmed the diagnosis of pelvic malignancy.

The role of neoadjuvant chemotherapy in overall survival rates of patients with ovarian cancer is controversial. The European Organization for Research and Treatment of Cancer (EORTC) attained clinical trial results (4) which support neoadjuvant chemotherapy in patients with ovarian cancer and demonstrated improvements in survival rates. However, the US GOG152 study and a small-scale British randomized controlled trial (RCT) $(4,5)$ demonstrated no benefit in survival rates. Previous joint study efforts of the EORTC and National Cancer Institute of Canada identified that neoadjuvant chemotherapy is no less effective than standard first-line treatment $(6,7)$. Nevertheless, in the present case neoadjuvant chemotherapy resulted in a favorable outcome. The metastatic lesions disappeared following 4 cycles of neoadjuvant chemotherapy. The patient's general condition improved significantly, which made interval cytoreductive surgery possible. An additional 3 courses of postoperative chemotherapy achieved complete clinical remission. Although relapse occurred 19 months after surgery, the disease was sensitive to platinum-based chemotherapy with a favorable prognosis. At this stage, the patient was enrolled in an international Phase III RCT for platinum-sensitive chemotherapy for recurrent cancer, where 4 cycles of combined liposomal doxorubicin and trabectidin second-line chemotherapy were administered. Once again, the disease was effectively controlled. Subsequently, the disease relapsed on multiple occasions and began developing resistance to chemotherapy. However, the patient's disease was controlled effectively for a long time with appropriate modifications to the drug regime. Eventually the patient succumbed 9 years after the primary diagnosis.

Once ovarian cancer relapses, the therapeutic modality changes from curative to palliative. Recurrent ovarian cancer, in the majority of instances, cannot be cured (3). However, palliative treatment may be effective in prolonging the survival rate and improving quality of life. In the past, platinum-sensitive chemotherapy for relapse involved the use of a single platinum-based agent. However, more recently, evidence-based medicine has revealed that a platinum-based combination therapy led to an improved outcome over monotherapy (8). On the basis of OVA-301 Phase III RCT results, platinum-containing combination agents including liposomal doxorubicin and trabectedin may significantly improve survival rates (8). The patient was enrolled in the OVA-301 study and benefited markedly with prolonged progression-free survival ( $>1$ year). Therefore, patients with ovarian cancer relapse may be encouraged to participate in clinical trials $(9,10)$. CA125 testing served a key role in disease follow-up. A previous Phase III RCT has confirmed that the initiation of chemotherapy at the onset of CA125 increase does not improve patients' long-term survival rates (8). Thus, a patient's CA125 level has a limited diagnostic role in the context of relapse and should always be supported by further imaging studies. Treatment should only be initiated upon confirmation of the diagnosis using imaging and physical examination (11).

In the present case of a patient with stage IV ovarian cancer, neoadjuvant chemotherapy, interval cytoreductive surgery and long-term multiple chemotherapy following surgery controlled the disease effectively. Multiple surgeries should not be considered as the standard treatment for recurrent ovarian cancer. Instead, multiple sessions of chemotherapy may be useful in the management of relapsed disease. Appropriate treatment should be selected on the basis of the patient's condition in order to maximize the therapeutic efficacy.

\section{References}

1. Jayson GC, Kohn EC, Kitchener HC and Ledermann JA: Ovarian cancer. Lancet 384: 1376-1388, 2014. 
2. Narod S: Can advanced-stage ovarian cancer be cured? Nat Rev Clin Oncol 13: 255-261, 2016.

3. Davidson B and Tropé CG: Ovarian cancer: Diagnostic, biological and prognostic aspects. Womens Health (Lond) 10: 519-533, 2014.

4. van der Burg ME, van Lent M, Buyse M, Kobierska A, Colombo N, Favalli G, Lacave AJ, Nardi M, Renard J and Pecorelli S: The effect of debulking surgery after induction chemotherapy on the prognosis in advanced epithelial ovarian cancer. Gynecological cancer cooperative group of the European organization for research and treatment of cancer. N Engl J Med 332: 629-634, 1995.

5. Redman CW, Warwick J, Luesley DM, Varma R, Lawton FG and Blackledge GR: Intervention debulking surgery in advanced epithelial ovarian cancer. Br J Obstet Gynaecol 101: 142-146, 1994.

6. Rose PG, Nerenstone S, Brady MF, Clarke-Pearson D, Olt G, Rubin SC, Moore DH and Small JM; Gynecologic Oncology Group: Secondary surgical cytoreduction for advanced ovarian carcinoma. N Engl J Med 351: 2489-2497, 2004.

7. Vergote I, Tropé CG, Amant F, Kristensen GB, Ehlen T, Johnson N, Verheijen RH, van der Burg ME, Lacave AJ, Panici PB, et al Neoadjuvant chemotherapy or primary surgery in stage IIIC or IV ovarian cancer. N Engl J Med 363: 943-953, 2010.
8. ColomboN: Efficacy of trabectedin in platinum-sensitive-relapsed ovarian cancer: New data from the randomized OVA-301 study. Int J Gynecol Cancer 21 (Suppl 1): S12-S16, 2011.

9. Kaye SB, Colombo N, Monk BJ, Tjulandin S, Kong B, Roy M, Chan S, Filipczyk-Cisarz E, Hagberg H, Vergote I, et al: Trabectedin plus pegylated liposomal doxorubicin in relapsed ovarian cancer delays third-line chemotherapy and prolongs the platinum-free interval. Ann Oncol 22: 49-58, 2011.

10. Poveda A, Vergote I, Tjulandin S, Kong B, Roy M, Chan S, Filipczyk-Cisarz E, Hagberg H, Kaye SB, Colombo N, et al: Trabectedin plus pegylated liposomal doxorubicin in relapsed ovarian cancer: Outcomes in the partially platinum-sensitive (platinum-free interval 6-12 months) subpopulation of OVA-301 phase III randomized trial. Ann Oncol 22: 39-48, 2011.

11. Rustin GJ: Follow-up with CA125 after primary therapy of advanced ovarian cancer has major implications for treatment outcome and trial performances and should not be routinely performed. Ann Oncol 22 (Suppl 8): viii45-viii48, 2011. 